\title{
Antiproliferative and proapoptotic effects of rapamycin and celecoxib in malignant melanoma cell lines
}

\author{
ANNIKA BUNDSCHERER* , CHRISTIAN HAFNER* , TIM MAISCH, \\ BERND BECKER, MICHAEL LANDTHALER and THOMAS VOGT \\ Department of Dermatology, University of Regensburg, D-93042 Regensburg, Germany
}

Received August 1, 2007; Accepted September 28, 2007

\begin{abstract}
Inhibitors of cyclooxygenase 2 (COX 2) and the mammalian target of rapamycin (mTOR) show direct and indirect antitumor effects in a variety of cancers. This study was designed to investigate the effects of the mTOR antagonist rapamycin and the COX 2 inhibitor celecoxib on cell growth and apoptosis in malignant melanoma. Cell proliferation was analysed by the cell proliferation ELISA BrdU and alamarBlue assay and apoptosis was measured by caspase 3 and 7 activity in two out of six melanoma cell lines (A375 and Mel Ho) that were selected for the heterogeneous levels of the COX 2 mRNA expression. The quantitative real-time reverse transcription polymerase chain reaction showed a 337-fold higher COX 2 mRNA level in the A375 than in the Mel Ho melanoma cells. However, both celecoxib and rapamycin caused significant growth inhibition in the two cell lines. By combining both agents, additive growth inhibitory effects were observed in the A375 cells. Treatment with celecoxib, but not rapamycin, increased apoptosis in the two cell lines. Our data indicate that rapamycin and celecoxib inhibit melanoma cell growth as single agents and a combination of both drugs have additive antitumor effects. Notably, the antiproliferative and proapoptotic effects of celecoxib seem to be independent of the COX 2 expression. Both rapamycin and celecoxib represent promising drugs for the palliative therapy of metastasised malignant melanoma and should be considered for future trials.
\end{abstract}

\section{Introduction}

In recent years, the incidence and mortality of malignant melanoma has continued to rise. While the early stages of the

Correspondence to: Dr Thomas Vogt, Department of Dermatology, University of Regensburg, Franz-Josef-Strauss-Allee 11, D-93042 Regensburg, Germany

E-mail: thomas.vogt@klinik.uni-regensburg.de

*Contributed equally

Key words: rapamycin, mammalian target of rapamycin, celecoxib, cyclooxygenase 2, malignant melanoma, growth inhibition, apoptosis, cell cycle arrest, combinatorial treatment disease can often be cured by appropriate therapy, advanced and metastasised malignant melanoma is characterised by a short median survival time and implies a poor prognosis. For this reason the treatment of advanced melanoma remains a challenge in dermatooncology. In the search for new strategies in tumor palliation, some well-established drugs such as rapamycin and celecoxib were shown to reveal broad antitumor activities (1) and, given their low toxicity their potential for palliative therapy of malignant melanoma seems to be grossly under-researched.

Rapamycin (sirolimus) is a natural fungicide isolated from the soil bacteria Streptomyces hygroscopius. Due to its profound immunosuppressive actions, rapamycin is used to prevent allograft rejection in organ transplantation. However, during its preclinical evaluation, rapamycin also revealed antitumor activities (2). As a complex combined with immunophilin FKBP 12, rapamycin inhibits the action of the mammalian target of rapamycin (mTOR), an atypical serine/threonine kinase playing a key role in the regulation of cell proliferation, differentiation, migration and survival (3). This kinase phosphorylates regulators of translation such as p70-S6 kinase and the eukaryotic translation initiation factor, $4 \mathrm{E}$ binding protein 1 (4E-BP1). Consequently, the blocking of mTOR results in cell cycle arrest, growth inhibition and a reduction of protein synthesis (3). The immunosuppressive potency of rapamycin is based on the inhibition of the lymphocyte proliferation stimulated by IL-2. The activity of mTOR is regulated by a concentration of amino acids, ATP and glucose. Activating growth factor signaling is transmitted via the IGFR-PI3K-AKT-mTOR pathway while TSC1/2 and PTEN are negative regulators of the mTOR function.

COX 1 and 2 are isoforms of the cyclooxygenase, a key enzyme in the conversion from arachidonic acid to prostaglandins. COX 1 is constitutively expressed in many tissues and catalyses the physiological prostaglandin synthesis. COX 2 is an immediate early response gene involved in pathological processes like inflammation and pain sensation. Under normal conditions, the COX 2 expression is low or not detectable in most tissues, but some exceptions such as the kidney and CNS are also known (4). COX 2 overexpression was found in a variety of human malignancies, including colorectal, head and neck, pancreatic, cervical, breast, gastric cancer (5) and malignant melanoma (6). In several clinical (7-11) and preclinical trials (12-15), NSAIDs and especially selective COX 2 inhibitors revealed anticancer activities. 
These effects are based on direct antitumor effects such as the induction of apoptosis and inhibition of tumor growth as well as on the modulation of the tumor-stroma interaction and inhibition of angiogenesis. Notably, some of the antitumor activities of COX 2 inhibitors seem to follow COX 2independent pathways $(12,15,16)$.

The aim of this study was to elucidate the antitumoral potential of rapamycin and the selective COX 2 inhibitor celecoxib administered singly or combined in malignant melanoma cells.

\section{Materials and methods}

Reagents and chemicals. Commercially available rapamycin (Rapamune $^{\circledR}$, Wyeth, Münster, Germany) was used as a stock solution. Celecoxib (Celebrex ${ }^{\circledR}$ ) was a generous gift from Pfizer. A stock solution of celecoxib was prepared by dissolving the drug in $100 \%$ dimethyl sulphoxide (DMSO). The final concentrations were achieved by diluting rapamycin and celecoxib stock solution in RPMI medium containing $10 \%$ fetal calf serum (FCS), penicillin-streptomycin (PS) and L-glutamine (LG) (Gibco). All solutions were prepared freshly prior to use. As celecoxib and rapamycin are bound to plasma proteins, the concentrations required for achieving antiproliferative effects in vitro are lower if the drugs are dissolved in serum-reduced or serum-free media. Since in vivo no serumfree conditions can be achieved, we decided to perform all the experiments with a medium containing $10 \%$ FCS.

Cell culture. Six human melanoma cell lines were screened for this study. A375 cells were purchased from the American Type Culture Collection and Mel Ho, Colo 849 and IGR 1 cells were purchased from the DSMZ (Deutsche Sammlung von Mikroorganismen und Zellkulturen). The nude-mouse selected isogenic variants of the A375 cell line with strong (SM) and poor (P) metastatic potential were kindly contributed by K. Gehlsen (S.K.C.C., San Diego, CA, USA) (17). A375, A375 P, A375 SM, Mel Ho and Colo 849 were grown in an RPMI-1640 formulation supplemented with $10 \%$ FCS $(20 \%$ for Colo 849), PS and $\mathrm{LG}$ (Gibco) at $37^{\circ} \mathrm{C}$ in a humified $\mathrm{CO}_{2}$ atmosphere (5 and 8\%). For IGR 1 cells, Dulbecco's modified Eagle's medium (DMEM) supplemented with 10\% FCS, PS and LG was used. Cells were cultured in Falcon $75-\mathrm{cm}^{2}$ tissue culture flasks and were maintained in a monolayer culture. Experiments were performed when cells reached $\sim 80 \%$ confluence.

RNA isolation and quantitative real-time reverse transcription polymerase chain reaction ( $R T-P C R)$. Six melanoma cell lines were screened for COX 2 mRNA expression. Cells were lysed by adding RLT buffer (Qiagen), released by scratching and lysates were homogenised by using Qia-shredder columns (Qiagen). RNA was extracted with the RNeasy Mini Kit (Qiagen) according to the manufacturer's protocol. RNA concentration was measured spectometrically at $260 \mathrm{~nm}$ and RNA was stored at $-80^{\circ} \mathrm{C}$. Total RNA was used as a template for cDNA synthesis according to the instructions of the Reverse Transcription System Kit (Promega). For quantification of the COX 2 transcripts TaqMan RT-PCR was performed as previously described $(18,19)$.
Cell proliferation. Proliferation of cells treated with celecoxib, rapamycin or the combination was determined using two different methods.

The alamarBlue $\mathrm{T}^{\mathrm{TM}}$ assay (Biosource) is a colorimetric growth indicator based on the detection of metabolic activity. Cells were seeded into 24-well plates (Falcon) at a cell density of $1.2 \times 10^{4}(\mathrm{~A} 375)$ or $1.5 \times 10^{4}(\mathrm{Mel} \mathrm{Ho})$ per well in the standard growth medium and were incubated for $24 \mathrm{~h}$ at $37^{\circ} \mathrm{C}$ to allow re-attachment. Then the culture medium was removed and replaced with $900 \mu 1$ of the test compounds (rapamycin, celecoxib or combination) or vehicle control (DMSO or RPMI) in various concentrations. AlamarBlue assay (100 $\mu \mathrm{l})$ was added into each well and the plates were cultured for $48 \mathrm{~h}$. After 24 and $48 \mathrm{~h}$ of treatment absorbance at 540 and $600 \mathrm{~nm}$ was measured using the Mithras multilabel reader (Berthold Technologies). All tests were performed in quadruplicate and repeated at least twice.

In addition, the BrdU (Roche) immunoassay was used to measure BrdU incorporation during DNA synthesis. Cells $\left(2-3 \times 10^{3}\right)$ were plated in 96-well plates (Costar) and incubated overnight at $37^{\circ} \mathrm{C}$. Then the cells were treated with $100 \mu 1$ of the test compounds and put back into the incubator for $32 \mathrm{~h}$ before being treated with $10 \mu \mathrm{l}$ of BrdU labelling solution for another $16 \mathrm{~h}$. After an overall treatment time with the test compounds of $48 \mathrm{~h}$, the medium was removed. The cells were incubated for 30 min with $200 \mu 1$ FixDenat solution at room temperature for fixing the cells and denaturating DNA and then for $90 \mathrm{~min}$ with antiBrdU-POD solution. Antibody conjugates were removed by washing three times with $200 \mu 1$ of washing buffer. After incubation with a TMB substrate for $15 \mathrm{~min}$, immune complexes were detected by measuring the absorbance at 405 and $490 \mathrm{~nm}$. Eight wells per treatment group were used and all tests were repeated at least twice. Drug concentration ranges were chosen according to the specific literature available $(13,20,21)$.

Apoptosis analysis. Cells $\left(10^{6}\right)$ were seeded into a $75-\mathrm{cm}^{2}$ tissue culture flask (Falcon) in the standard growth medium and were allowed to adhere for $24 \mathrm{~h}$ before being treated with celecoxib $(50 \mu \mathrm{M})$ and/or rapamycin $(0.5 \mu \mathrm{M})$ or vehicle (DMSO or RPMI) for $24 \mathrm{~h}$. For apoptosis analysis, the activity of caspase 3 and 7 was measured by a Caspase-Glo ${ }^{\text {TM }} 3$ and 7 assay (Promega). The cells were released by scratching and re-suspended in serum-free medium, counted and seeded into a white 96 -well multititer plate $\left(10^{4}\right.$ cells in $100 \mu 1$ serum-free media/well). Eight wells per treatment group were used. After equilibration to room temperature, $100 \mu \mathrm{l}$ of CaspaseGlo 3 and 7 assay were added to each well. The plate was incubated at room temperature and protected from light for $1 \mathrm{~h}$. Finally, luminescence was measured using the Mithras multilabel reader and Mikro-Win Software.

Cell cycle analysis. Cell cycle profiles were measured by flow cytometry. A375 and Mel Ho cells were cultured with celcoxib $(50 \mu \mathrm{M})$ and/or rapamycin $(0.5 \mu \mathrm{M})$ or vehicle for $24 \mathrm{~h}$. The cells were released by treatment with trypsin-EDTA, counted ( $10^{6}$ cells), washed twice with cold PBS/5 mM EDTA and fixed at room temperature with $100 \%$ ethanol for $30 \mathrm{~min}$. After washing again with PBS/5 mM EDTA, the cells were treated with $1 \mathrm{mg} / \mathrm{ml}$ RNase A and incubated for another 
$30 \mathrm{~min}$ at room temperature. Finally, the cells were stained with $100 \mu \mathrm{g} / \mathrm{ml}$ propidium iodide (Sigma) and analysed by flow cytometry using FACSCalibur and Cellquest software. For each sample $10^{4}$ fluorescent cells were counted.

Statistical analysis. The results are expressed as means \pm SD. For comparisons between the means, the non-parametric Mann-Whitney U test was applied. Cell proliferation was calculated relative to the untreated control which was designated at $100 \%$, whereas the data obtained by apoptosis and cell cycle analysis were compared directly. For this reason, the statistical tests were 1 -sided for the cell proliferation experiments and 2-sided for the apoptosis and cell cycle analysis. Differences were considered statistically significant at $\mathrm{p}<0.05$.

\section{Results}

COX 2 mRNA expression in A375 and Mel Ho melanoma cell lines. To analyse a possible dependence of the COX 2 expression on the effectiveness of coxibs we first screened six melanoma cell lines (A375, A375 SM, A375 P, Mel Ho, IGR 1 and Colo 849) for their COX 2 mRNA expression level using the quantitative RT-PCR (Fig. 1). The COX 2 expression was detectable in all melanoma cell lines. The COX 2 mRNA level was highest in A375, followed by A375 SM and Colo 849 cell lines. In the IGR 1, A375 P and Mel Ho melanoma cell lines COX 2 expression was very low. To determine whether the effects of the COX 2 inhibitors were influenced by the COX 2 expression, we selected A375 (high expression) and Mel Ho (low expression) melanoma cell lines for further investigation. In the A375 cells the COX 2 mRNA expression
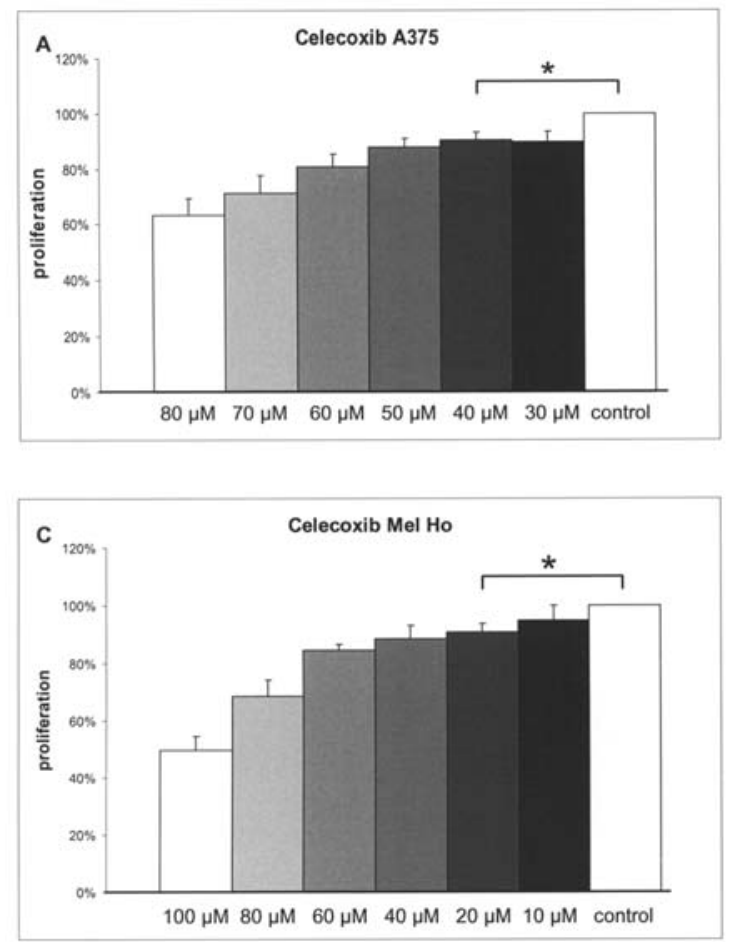

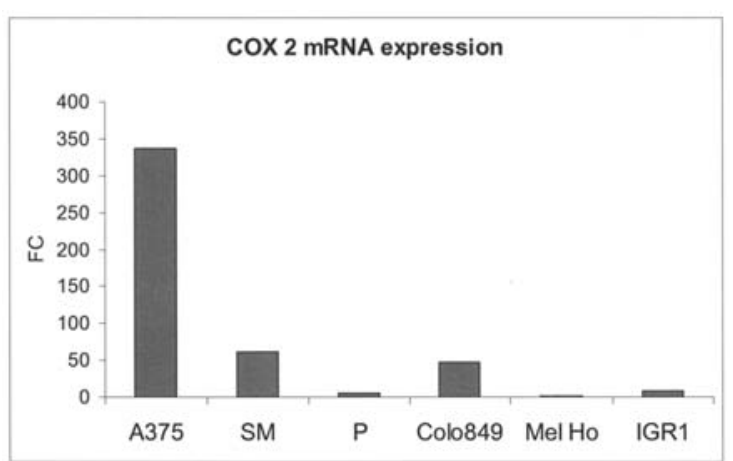

Figure 1. Quantitative RT-PCR for COX 2 baseline expression in six melanoma cell lines. COX 2 was expressed in all melanoma cell lines. The COX 2 expression in the A375 cells is 337-fold higher than in the Mel Ho melanoma cells. The COX 2 mRNA expression of Mel Ho cells served as the calibrator (value $=1)$, $(\mathrm{FC}$, fold change) .

was 337-fold higher than in the Mel Ho melanoma cells. A similar analysis for mTOR was not possible since no quantitative tool is currently available.

Celecoxib and rapamycin inhibit proliferation in melanoma cell lines. The A375 and Mel Ho cell lines were incubated with increasing concentrations of rapamycin $(0.2-1 \mu \mathrm{M})$, celecoxib $(10-100 \mu \mathrm{M})$ or a combination of both drugs $(50 \mu \mathrm{M}$ celecoxib and $0.5 \mu \mathrm{M}$ rapamycin) for $48 \mathrm{~h}$. The effects on cell proliferation were measured by the alamarBlue assay, a colorimetric growth indicator based on the detection of metabolic activity. Celecoxib caused a significant, dosedependent inhibition of proliferation in both cell lines (Fig. 2A and $\mathrm{B}$ ). For example, $80 \mu \mathrm{M}$ celecoxib significantly reduced
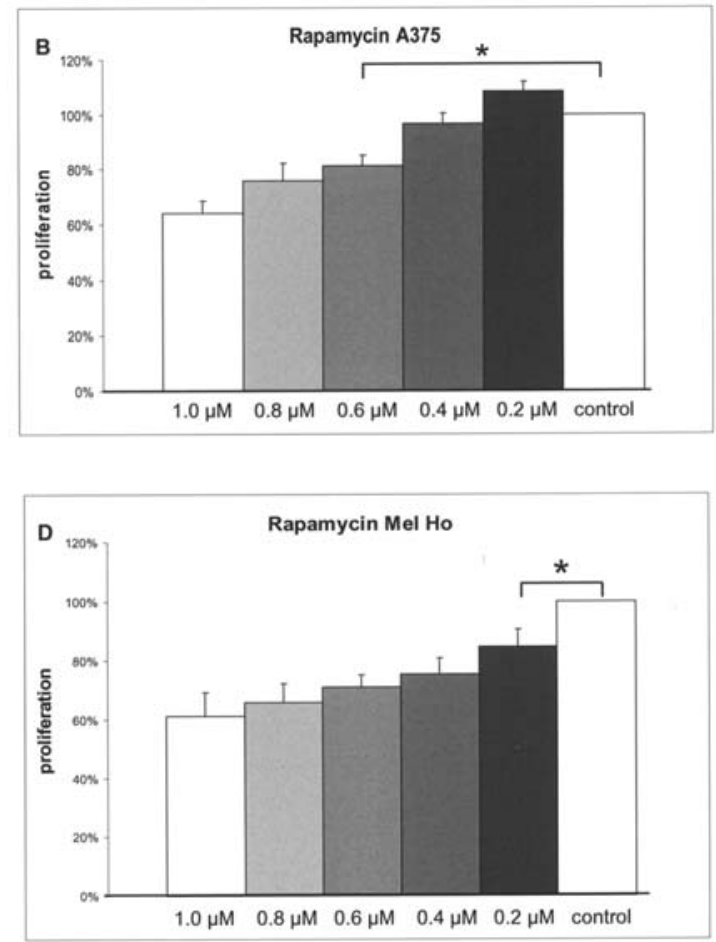

Figure 2. Dose-dependent growth inhibition by celecoxib and rapamycin. Mel Ho and A375 melanoma cells were grown overnight in a 24-well plate and then treated with increasing concentrations of celecoxib and rapamycin for $48 \mathrm{~h}$. Cell proliferation was measured using the alamarBlue assay and was calculated relative to the untreated control (DMSO for celecoxib and standard growth medium for rapamycin-treated cells). (A) A375 treated with celecoxib, (B) Mel Ho treated with celecoxib, (C) A375 treated with rapamycin and (D) Mel Ho treated with rapamycin; *p<0.001 compared with the respective control culture. 

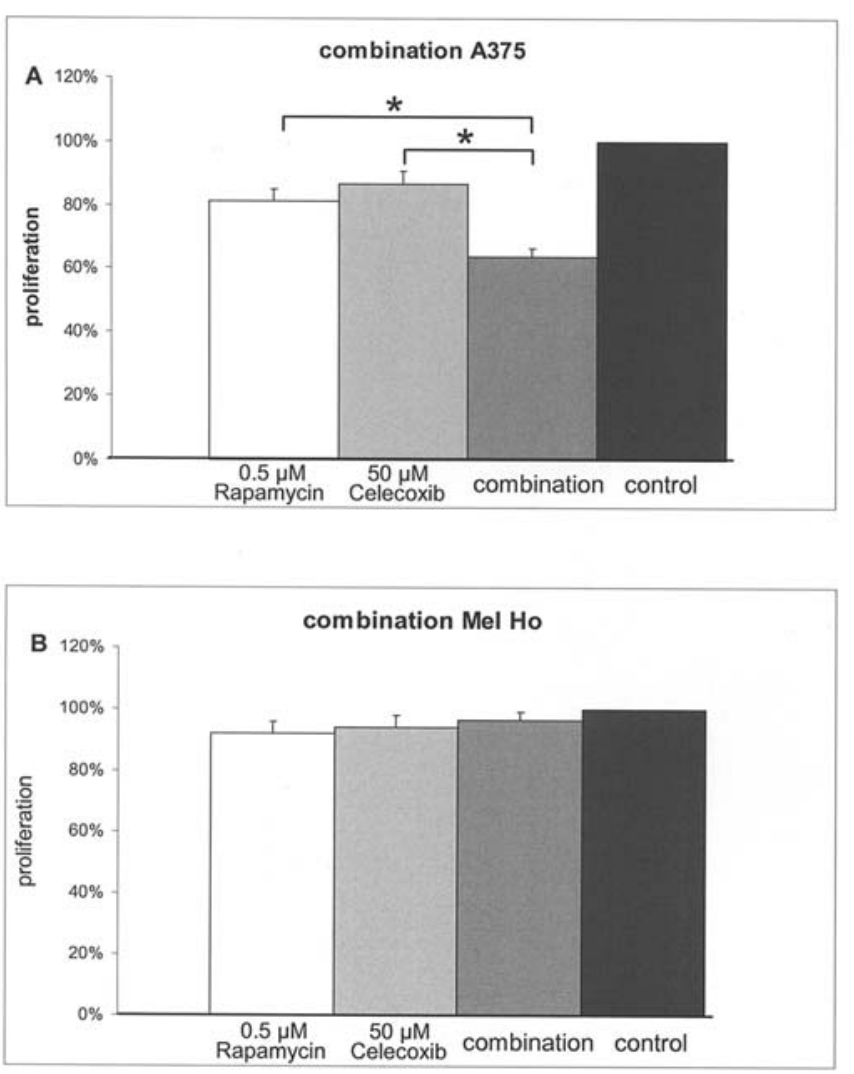

Figure 3. Combinatorial treatment with rapamycin and celecoxib. A375 (A) and Mel Ho (B) melanoma cell lines were treated with rapamycin $(0.5 \mu \mathrm{M})$, celecoxib $(50 \mu \mathrm{M})$ or a combination of both agents for $48 \mathrm{~h}$. Cell proliferation was measured using the alamarBlue assay and was calculated relative to the untreated control (DMSO for the celecoxib and combination and a standard growth medium for rapamycin-treated cells); $\mathrm{p}=0.002$ compared with the respective control.

cell growth (by $31 \pm 6$ in Mel Ho and $37 \pm 6 \%$ in A375). Thus the antiproliferative effects were comparable in the two melanoma cell lines regardless of the difference in the COX 2 expression level. Treatment with rapamycin also resulted in a dose-dependent growth inhibition. Rapamycin $(1 \mu \mathrm{M})$ reduced A375 and Mel Ho cell proliferation by $36 \pm 4$ and $39 \pm 8 \%$, respectively (Fig. 2C and D). At lower concentrations, targeting the mTOR was more effective in the Mel Ho than in the A375 melanoma cell line. To confirm the data obtained by the alamarBlue assay, we used the cell proliferation ELISA BrdU assay. We detected very similar growth inhibitory effects (data not shown). In several preclinical studies mTOR antagonists and COX 2 inhibitors showed encouraging results when used in combination with other anticancer agents. For this reason we were interested in whether a combinatorial treatment of rapamycin and celecoxib would exert stronger antiproliferative effects on melanoma cell proliferation than each drug alone. To determine potential additive or even super-additive effects, we chose drug concentrations which only caused slight growth inhibition when administered as single agents. By combining $50 \mu \mathrm{M}$ celecoxib with $0.5 \mu \mathrm{M}$ rapamycin, a significant increase in growth inhibition was detected in the A375 cells (Fig. 3A). While rapamycin and celecoxib reduced melanoma cell proliferation by $19 \pm 8$ and $13 \pm 6 \%$ respectively, the combinatorial
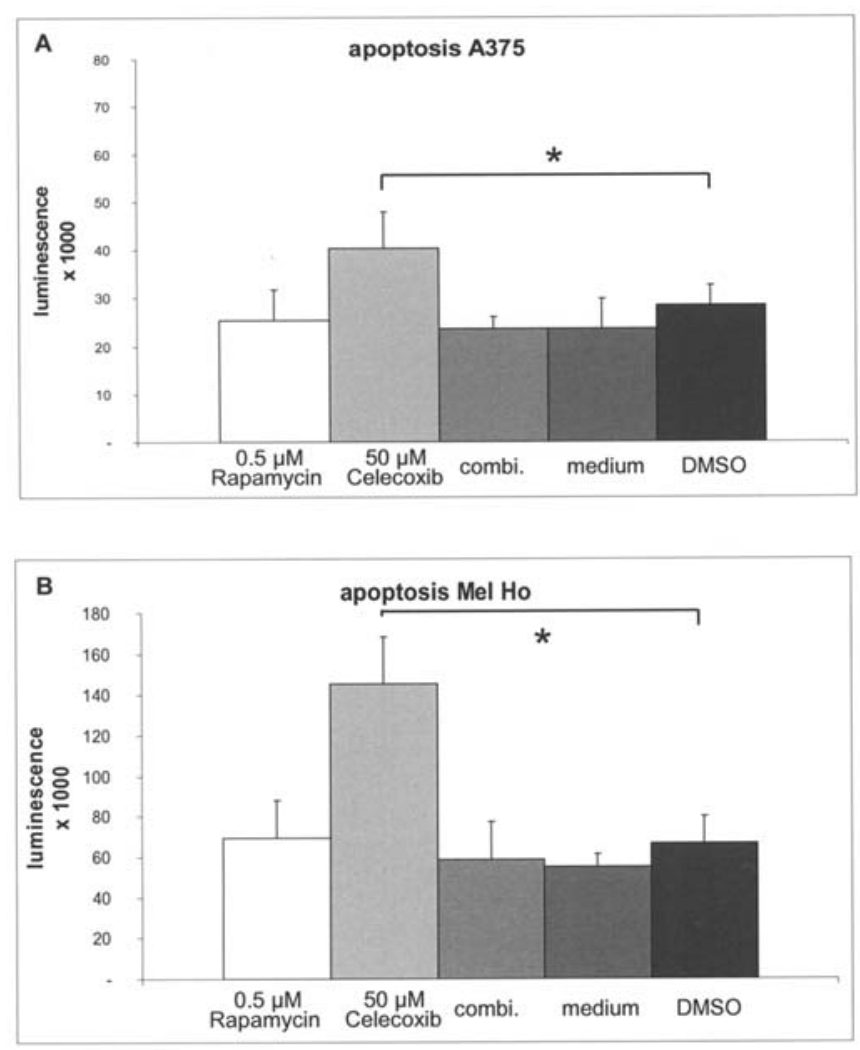

Figure 4. Induction of apoptosis in A375 (A) and Mel Ho (B) melanoma cells. Cells were treated for $24 \mathrm{~h}$ with rapamycin $(0.5 \mu \mathrm{M})$, celecoxib $(50 \mu \mathrm{M})$, a combination (rapamycin $0.5+$ celecoxib $50 \mu \mathrm{M}$ ) or a medium as the untreated control for rapamycin and DMSO as the untreated control for celecoxib and the combination. The activity of caspase 3 and 7 was analysed using the Caspase-Glo 3 and 7 assay. Luminescence values correlate directly with caspase activity; ${ }^{*} \mathrm{p}<0.001$.

treatment inhibited proliferation at a significantly higher rate $(36 \pm 4 \%)(\mathrm{p}=0.002)$. In the Mel Ho cells no additive growth inhibitory effects were detected (Fig. 3B).

Celecoxib induces apoptosis in melanoma cell lines. In order to ascertain if the inhibition of cell proliferation is associated with an enhanced apoptosis rate, we measured the activity of caspase 3 and 7 after incubation using the same concentration as mentioned above, i.e., $0.5 \mu \mathrm{M}$ rapamycin and/or $50 \mu \mathrm{M}$ celecoxib for $24 \mathrm{~h}$ (Fig. 4). Celecoxib significantly increased caspase activity in the A375 and Mel Ho cell lines by 1.4- and 2.2 -fold, respectively, when compared with the untreated vehicle control. Rapamycin caused a slight enhancement of apoptosis, which only reached a significant level in the Mel Ho cells (1.1- and 1.3-fold, respectively). By combining rapamycin and celecoxib, a modest but significant protection against apoptosis were observed in the two cell lines.

Rapamycin but not celecoxib induces cell cycle arrest. To elucidate the mechanism underlying the growth inhibition, we performed a cell cycle analysis. In the A375 cells rapamycin caused an increase in cells in the G-1 phase from $56.5 \pm 4.1$ to $67.0 \pm 2.0 \%$ compared to the untreated control, accompanied by a decrease in the G-2 phase from $31.4 \pm 1.3$ to $23.4 \pm 1.9 \%$ (Fig. 5). In Mel Ho the increase from 56.5 \pm 3.5 to $59.5 \pm 2.1 \%$ 
Table I. Cell cycle analysis.

\begin{tabular}{|c|c|c|c|c|c|c|}
\hline & \multicolumn{3}{|c|}{ Mel Ho } & \multicolumn{3}{|c|}{ A375 } \\
\hline & G-1 & $S$ & G-2 & G-1 & $\mathrm{S}$ & G-2 \\
\hline Medium & $56.5 \pm 3.7$ & $15.7 \pm 1.2$ & $25.6 \pm 2.6$ & $56.5 \pm 4.1$ & $11.9 \pm 3.2$ & $31.4 \pm 1.3$ \\
\hline Rapamycin $(0.5 \mu \mathrm{M})$ & $59.5 \pm 2.1$ & $10.6 \pm 0.0$ & $24.6 \pm 1.9$ & $67.0 \pm 2.0$ & $9.6 \pm 2.1$ & $23.4 \pm 1.9$ \\
\hline DMSO & $58.0 \pm 1.1$ & $14.6 \pm 1.0$ & $24.7 \pm 0.5$ & $61.6 \pm 2.0$ & $9.9 \pm 0.0$ & $26.5 \pm 1.8$ \\
\hline Celecoxib $(50 \mu \mathrm{M})$ & $62.9 \pm 5.4$ & $11.2 \pm 0.6$ & $22.8 \pm 4.8$ & $60.3 \pm 1.9$ & $11.2 \pm 0.9$ & $27.0 \pm 2.1$ \\
\hline Combination & $61.7 \pm 3.8$ & $11.3 \pm 0.4$ & $22.2 \pm 1.6$ & $62.1 \pm 2.5$ & $11.8 \pm 1.1$ & $22.6 \pm 1.7$ \\
\hline
\end{tabular}

Cell cycle distribution of the Mel Ho and A375 cells after treatment for $24 \mathrm{~h}$ with rapamycin $(0.5 \mu \mathrm{M})$, celecoxib $(50 \mu \mathrm{M})$, a combination or control (medium for rapamycin and DMSO for celecoxib-treated cells). Data are presented as means $(\%) \pm$ SD.

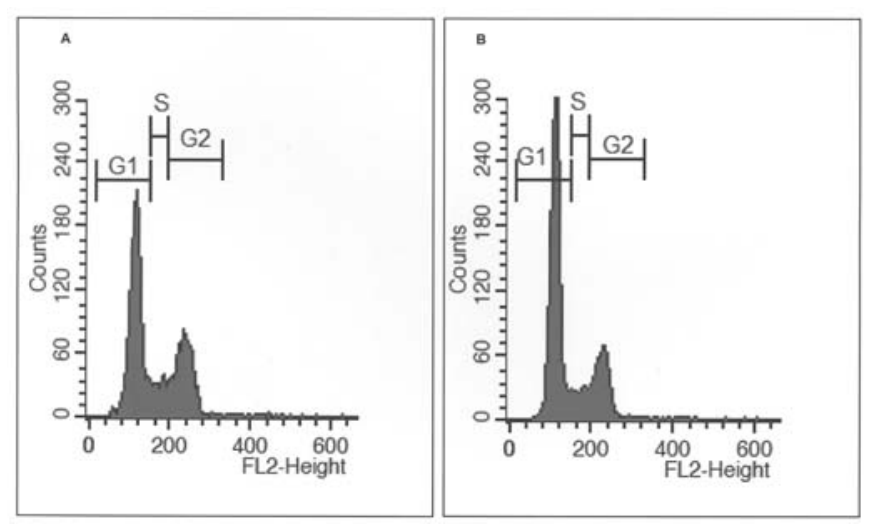

Figure 5. Rapamycin induces cell cycle arrest in A375 melanoma cells. Representative DNA histogram of the cells after treatment with the medium (A) or $0.5 \mu \mathrm{M}$ rapamycin (B) for $24 \mathrm{~h}$. The nuclei were stained with propidium iodide and cell cycle distribution was detected by flow cytometry.

induced by rapamycin was not significant. In the melanoma cells treated with celecoxib and a combination of both agents only slight changes in the cell cycle could be detected (Table I).

\section{Discussion}

In this study we demonstrated that celecoxib and rapamycin inhibit melanoma cell proliferation in a dose-dependent fashion and that celecoxib but not rapamycin induce significant apoptosis. The growth inhibitory effects in celecoxib seem to be independent of the COX 2 baseline expression in the melanoma cells. A combinatorial treatment with both agents resulted in the additive inhibition of cell growth in the A375 melanoma cells, although this effect was accompanied by a decrease of caspase activity.

COX 2 is involved in carcinogenesis and promotes tumor cell invasion and metastasis. COX 2 overexpression was found in a variety of human malignancies, including colorectal, head and neck, pancreatic, cervical, breast, gastric cancer (5) and malignant melanoma (6). In this study, COX 2 mRNA was differentially expressed in six melanoma cell lines. The significance of the COX 2 overexpression in melanoma pathogenesis is still controversial, but the expression seems to vary considerably in malignant melanoma and metastasised malignant melanoma $(6,16,22)$. This raised the question of whether low COX 2-expressing cell lines would be less susceptible to coxibs. To answer this question, we chose A375 as high and Mel Ho as low COX 2-expressing cell lines for further investigation. In both cell lines, celecoxib inhibited proliferation in a dose-dependent fashion and induced apoptosis by enhancing the caspase 3 and 7 activity. The growth inhibitory effects were comparable in the two cell lines and the enhancement of the caspase 3 and 7 activity was stronger in the Mel Ho melanoma cells. These data indicate that the anticancer effects of celecoxib do not depend on the COX 2 expression in melanoma. These findings agree with the results of a previous study comparing the effects of sulindac sulphide on the apoptosis of COX 2-negative melanoma and COX 2-positive colon carcinoma cells. As there was no significant difference in the rate of apoptotic cell death, NSAID-induced apoptosis was judged to follow COX 2 independent pathways (16). Similar results were obtained in several tumor entities by investigating either COX 2-deficient cell lines $(12,15)$, cell lines with a low baseline COX 2 expression (21) or silencing COX 2 activity by antisense depletion (13). In addition, it was reported that NSAIDs reveal their antitumor potency only at doses that are $>100$ - to 1000 -fold than those required to block the prostaglandin synthesis by inhibition of the COX 2 activity (23).

In this study we detected the inhibition of cell proliferation with a broad range of concentrations of celecoxib (10 to $100 \mu \mathrm{M}$ ). Regarding apoptosis, a concentration of $50 \mu \mathrm{M}$ of celecoxib induced an enhancement of caspase 3 and 7 activity in the two cell lines while no changes in cell cycle distribution could be measured. The induction of apoptosis and cell cycle arrest are effects often observed in cells treated with celecoxib, whereas the concentrations required to achieve these effects vary among different cancer types $(12,13,15)$. Reportedly, some mechanisms underlying coxib-induced apoptosis have already been defined. Dandekar et al observed an augmentation of chemotherapeutic-drug-induced apoptosis in prostate cancer cells via the activation of caspase 3 and 9, decreased levels of Bcl-xl and increased BAD concentration (24). In non-small cell lung cancer celecoxibtriggered apoptosis was shown to be based on the activation of death receptor 5 (25). Furthermore, a reduction of protein kinase B/AKT signaling was described after treatment with celecoxib (26). In melanoma the induction of FasL-mediated 
death signaling by coxibs was observed (27). In the clinical trials celecoxib in combination with other anticancer agents showed encouraging results without causing significant toxicities (7-11).

Rapamycin has emerged as a more recent biomodulator in cancer. Therefore, we further investigated the effects of mTOR antagonists on the proliferation, apoptosis and cell cycle of those melanoma cell lines. The mTOR-antagonist rapamycin was effective in inhibiting melanoma cell proliferation in a dose-dependent fashion. Treatment with $1 \mu \mathrm{M}$ rapamycin reduced A375 and Mel Ho cell growth by 36 and 39\%, respectively. While similar results were found in mammary tumors (20), in other cancers even nanomolar concentrations of rapamycin lead to comparable antiproliferative effects $(28,29)$. Some investigators have postulated that targeting the mTOR may be most potent in tumors containing PTEN mutations.

In melanoma, a loss of the PTEN function is a late step in melanoma tumorigenesis. Accordingly, a PTEN-mutation rate of $30-50 \%$ was detected in melanoma cell lines whereas only $10 \%$ of primary melanomas exhibit PTEN alterations (30). In the A375 cell line no alterations in the PTEN status were found (31). This may explain the high doses of rapamycin required to achieve growth inhibition in this particular melanoma cell line in comparison with other tumor types. The PTEN status of Mel Ho has yet to be determined.

In addition to the antiproliferative effects, $0.5 \mu \mathrm{M}$ rapamycin induced a certain degree of arrest in the G-1 phase of the cell cycle in the A375 cells plus a modest enhancement of the caspase 3 and 7 activity. Our findings partly confirm other preclinical studies showing an increasing number of cells in the G-1 phase of the cell cycle by the up-regulation of p21, p27 and CDK1 together with the down-regulation of cyclin $\mathrm{A}$ and cyclin $\mathrm{D}_{1} / \mathrm{CDK} 4$ levels (28). Accordingly, rapamycin blocked cell cycle progression without inducing apoptotic cell death in renal and non-small cell lung cancer $(32,33)$. Some investigators described a correlation between an enhanced bcl-2 expression or activation of AKT in cancer cells and protection against rapamycin-induced apoptosis $(34,35)$. In contrast, in anaplastic large cell lymphoma rapamycin enhanced apoptosis by decreasing the antiapoptotic molecules BCL-2, BCL-xl and c-FLIP in a dose-dependent manner (28). The modest enhancement of the apoptosis rate by rapamycin in melanoma cell lines may be based on similar mechanisms.

Although some clinical trials using mTOR antagonists in patients with advanced cancers showed encouraging results, in malignant melanoma a monotherapy with the rapamycin analogue CCI-779 was not effective. In a phase-2 trial of the California Cancer Consortium only 1 out of 33 patients with metastatic melanoma had a partial response (36). However, a combinatorial treatment with rapamycin and other antitumor agents such as doxorubicin or the B-Raf-inhibitor BAY439006 resulted in an enhanced antitumor potential in melanoma in vitro $(37,38)$. Furthermore, in several preclinical studies celecoxib has been combined with cyotoxic or biomudulating agents and revealed synergistic anticancer activities $(24,39)$. To our knowledge, the effects of a combinatorial treatment with celecoxib and rapamycin on melanoma cell proliferation have not previously been examined. We hypothesized that a combination of the two agents would reveal synergistic antitumor activities. In the A375 cells celecoxib and rapamycin synergistically reduced cell growth, but no additive effects could be detected in the Mel Ho cells. Since the A375 and Mel Ho cells differ in their COX 2 baseline expression, these data indicate that the efficiency of the rapamycin-celecoxib combination may depend on the COX 2 expression levels. These findings agree with a study by El-Rayes et al who postulated that a low COX 2 baseline expression may diminish the benefits from a combinatorial treatment with celecoxib and gemcitabine (21). Notably, the inhibition of growth is not necessarily limited to apoptosis as rapamycin seems to protect the melanoma cells from celecoxib-induced apoptosis. The results of several preclinical studies indicated that the effects on the apoptosis rate caused by a combinatorial treatment depend on the cytotoxic agents that the mTOR inhibitors are combined with. In multiple myeloma cells rapamycin inhibited apoptosis induced by PS-341 (35) but sensitized to dexamethasone (29). Furthermore, rapamycin protected B-cell lymphoma cells from etopside- and paclitaxel-induced caspase activation whereas apoptosis by doxorubicin was not affected (34). Romano et al even described an enhancement of the cytolytic effects of doxorubicin in melanoma cells (38). Combinations of celecoxib and chemotherapeutic agents or the tyrosine kinase inhibitor emodin resulted in an augmentation of apoptotic cell death $(24,40)$.

In conclusion, a potential benefit of the combinatorial use of coxibs and mTOR inhibitors emerges that may be even more pronounced if stroma-targeting antitumor effects such as antiangiogenesis are taken into account. These effects need to be determined in future experimental studies.

\section{Acknowledgements}

We are grateful to Pfizer for providing the celecoxib as a gift and to Lydia Kuenzel and Andrea Mueller for technical support.

\section{References}

1. Hafner C, Reichle A and Vogt T: New indications for established drugs: combined tumor-stroma-targeted cancer therapy with PPARgamma agonists, COX 2 inhibitors, mTOR antagonists and metronomic chemotherapy. Curr Cancer Drug Targets 5: 393-419, 2005

2. Eng CP, Sehgal SN and Vezina C: Activity of rapamycin (AY22,989) against transplanted tumors. J Antibiot 37: 1231-1237, 1984

3. Huang S and Houghton PJ: Targeting mTOR signaling for cancer therapy. Curr Opin Pharmacol 3: 371-377, 2003.

4. Zha S, Yegnasubramanian V, Nelson WG, Isaacs WB and De Marzo AM: Cyclooxygenases in cancer: progress and perspective. Cancer Lett 215: 1-20, 2004.

5. Sanborn R and Blanke CD: Cyclooxygenase 2 inhibition in colorectal cancer: boom or bust? Semin Oncol 32: 69-75, 2005.

6. Denkert C, Kobel M, Berger S, Siegert A, Leclere A, Trefzer U and Hauptmann S: Expression of cyclooxygenase 2 in human malignant melanoma. Cancer Res 61: 303-308, 2001.

7. Vogt T, Coras B, Hafner C, Landthaler M and Reichle A: Antiangiogenetic and anti-inflammatory palliative therapy in case of metastatic prostate carcinoma complicated by paraneoplastic subacute-cutaneous lupus erythemathodes. Lancet Oncol (In press).

8. Vogt T, Hafner C, Bross K, Bataille F, Jauch KW, Berand A, Landthaler M, Andreesen R and Reichle A: Antiangiogenetic therapy with pioglitazone, rofecoxib and metronomic trofosfamide in patients with advanced malignant vascular tumors. Cancer 98: 2251-2256, 2003. 
9. Coras B, Hafner C, Reichle A, Hohenleutner U, Szeimies RM, Landthaler $M$ and Vogt T: Antiangiogenic therapy with pioglitazone, rofecoxib, and trofosfamide in a patient with endemic kaposi sarcoma. Arch Dermatol 140: 1504-1507, 2004.

10. Reichle A, Bross K, Vogt T, Bataille F, Wild P, Berand A, Krause SW and Andreesen R: Pioglitazone and rofecoxib combined with angiostatically scheduled trofosfamide in the treatment of far-advanced melanoma and soft tissue sarcoma. Cancer 101: 2247-2256, 2004.

11. Reichle A, Vogt T, Kunz-Schughart L, Bretschneider T, Bachthaler M, Bross K, Freund S and Andreesen R: Antiinflammatory and angiostatic therapy in chemorefractory multisystem Langerhans' cell histiocytosis of adults. Br J Haematol 128: 730-732, 2005

12. Patel MI, Subbaramaiah K, Du B, Chang M, Yang P, Newman RA, Cordon-Cardo C, Thaler HT and Dannenberg AJ: Celecoxib inhibits prostate cancer growth: evidence of a cyclooxygenase 2-independent mechanism. Clin Cancer Res 11: 1999-2007, 2005.

13. Han C, Leng J, Demetris AJ and Wu T: Cyclooxygenase 2 promotes human cholangiocarcinoma growth: evidence for cyclooxygenase 2 -independent mechanism in celecoxibmediated induction of p21waf1/cip1 and p27kip1 and cell cycle arrest. Cancer Res 64: 1369-1376, 2004.

14. Kim SH, Song SH, Kim SG, Chun KS, Lim SY, Na HK, Kim JW, Surh YJ, Bang YJ and Song YS: Celecoxib induces apoptosis in cervical cancer cells independent of cyclooxygenase using $\mathrm{NF}-\kappa \mathrm{B}$ as a possible target. J Cancer Res Clin Oncol 130: 551-560, 2004.

15. Waskewich C, Blumenthal RD, Li H, Stein R, Goldenberg DM and Burton J: Celecoxib exhibits the greatest potency amongst cyclooxygenase (COX) inhibitors for growth inhibition of COX 2-negative hematopoietic and epithelial cell lines. Cancer Res 62: 2029-2033, 2002

16. Vogt T, McClelland M, Jung B, Popova S, Bogenrieder T, Becker B, Rumpler G, Landthaler M and Stolz W: Progression and NSAID-induced apoptosis in malignant melanomas are independent of cyclooxygenase 2. Melanoma Res 11: 587-599, 2001.

17. Gehlsen KR, Davis GE and Sriramarao P: Integrin expression in human melanoma cells with differing invasive and metastatic properties. Clin Exp Metastasis 10: 111-120, 1992.

18. Hafner C, Schmitz G, Meyer S, Bataille F, Hau P, Langmann T, Dietmaier W, Landthaler M and Vogt T: Differential gene expression of Eph receptors and ephrins in benign human tissues and cancers. Clin Chem 50: 490-499, 2004

19. Langmann T, Mauerer R, Zahn A, Moehle C, Probst M, Stremmel W and Schmitz G: Real-time reverse transcriptionPCR expression profiling of the complete human ATP-binding cassette transporter superfamily in various tissues. Clin Chem 49: 230-238, 2003.

20. Namba R, Young LJ, Abbey CK, Kim L, Damonte P, Borowsky AD, Qi J, Tepper CG, MacLeod CL, Cardiff RD and Gregg JP: Rapamycin inhibits growth of premalignant and malignant mammary lesions in a mouse model of ductal carcinoma in situ. Clin Cancer Res 12: 2613-2621, 2006.

21. El-Rayes BF, Ali S, Sarkar FH and Philip PA: Cyclooxygenase 2-dependent and -independent effects of celecoxib in pancreatic cancer cell lines. Mol Cancer Ther 3: 1421-1426, 2004.

22. Goulet AC, Einsphar JG, Alberts DS, Beas A, Burk C, Bhattacharyya A, Bangert J, Harmon JM, Fujiwara H, Koki A and Nelson MA: Analysis of cyclooxygenase 2 (COX 2) expression during malignant melanoma progression. Cancer Biol Ther 2: 713-718, 2003.

23. Tegeder I, Pfeilschifter J and Geisslinger G: Cyclooxygenaseindependent actions of cyclooxygenase inhibitors. FASEB J 15: 2057-2072, 2001.

24. Dandekar DS, Lopez M, Carey RI and Lokeshwar BL: Cyclooxygenase 2 inhibitor celecoxib augments chemotherapeutic drug-induced apoptosis by enhancing activation of caspase- 3 and -9 in prostate cancer cells. Int J Cancer 115 . 484-492, 2005
25. Liu X, Yue P, Zhou Z, Khuri FR and Sun SY: Death receptor regulation and celecoxib-induced apoptosis in human lung cancer cells. J Natl Cancer Inst 96: 1769-1780, 2004.

26. Kulp SK, Yang YT, Hung CC, Chen KF, Lai JP, Tseng PH, Fowble JW, Ward PJ and Chen CS: 3-phosphoinositidedependent protein kinase-1/Akt signaling represents a major cyclooxygenase 2-independent target for celecoxib in prostate cancer cells. Cancer Res 64: 1444-1451, 2004.

27. Ivanov VN and Hei TK: Dual treatment with COX 2 inhibitor and sodium arsenite leads to induction of surface Fas Ligand expression and Fas-Ligand-mediated apoptosis in human melanoma cells. Exp Cell Res (In press).

28. Vega F, Medeiros LJ, Leventaki V, Atwell C, Cho-Vega JH, Tian L, Claret FX and Rassidakis GZ: Activation of mammalian target of rapamycin signaling pathway contributes to tumor cell survival in anaplastic lymphoma kinase-positive anaplastic large cell lymphoma. Cancer Res 66: 6589-6597, 2006.

29. Yan H, Frost P, Shi Y, Hoang B, Sharma S, Fisher M, Gera J and Lichtenstein A: Mechanism by which mammalian target of rapamycin inhibitors sensitize multiple myeloma cells to dexamethasone-induced apoptosis. Cancer Res 66: 2305-2313, 2006.

30. Wu H, Goel V and Haluska FG: PTEN signaling pathways in melanoma. Oncogene 22: 3113-3122, 2003.

31. Tsao H, Zhang X, Fowlkes K and Haluska FG: Relative reciprocity of NRAS and PTEN/MMAC1 alterations in cutaneous melanoma cell lines. Cancer Res 60: 1800-1804, 2000.

32. Luan FL, Ding R, Sharma VK, Chon WJ, Lagman M and Suthanthiran M: Rapamycin is an effective inhibitor of human renal cancer metastasis. Kidney Int 63: 917-926, 2003.

33. Boffa DJ, Luan F, Thomas D, Yang H, Sharma VK, Lagman M and Suthanthiran M: Rapamycin inhibits the growth and metastatic progression of non-small cell lung cancer. Clin Cancer Res 10: 293-300, 2004

34. Calastretti A, Rancati F, Ceriani MC, Asnaghi L, Canti G and Nicolin A: Rapamycin increases the cellular concentration of the BCL-2 protein and exerts an anti-apoptotic effect. Eur J Cancer 37: 2121-2128, 2001

35. Shi Y, Yan H, Frost P, Gera J and Lichtenstein A: Mammalian target of rapamycin inhibitors activate the AKT kinase in multiple myeloma cells by up-regulating the insulin-like growth factor receptor/insulin receptor substrate-1/phosphatidylinositol 3-kinase cascade. Mol Cancer Ther 4: 1533-1540, 2005.

36. Margolin K, Longmate J, Baratta T, Synold T, Christensen S, Weber J, Gajewski T, Quirt I and Doroshow JH: CCI-779 in metastatic melanoma: a phase II trial of the California Cancer Consortium. Cancer 104: 1045-1048, 2005.

37. Molhoek KR, Brautigan DL and Slingluff CL Jr: Synergistic inhibition of human melanoma proliferation by combination treatment with B-Raf inhibitor BAY43-9006 and mTOR inhibitor Rapamycin. J Transl Med 3: 39, 2005.

38. Romano MF, Avellino R, Petrella A, Bisogni R, Romano S and Venuta S: Rapamycin inhibits doxorubicin-induced NF-кB/Rel nuclear activity and enhances the apoptosis of melanoma cells. Eur J Cancer 40: 2829-2836, 2004.

39. Chiu LC, Tong KF and Ooi VE: Cytostatic and cytotoxic effects of cyclooxygenase inhibitors and their synergy with docosahexaenoic acid on the growth of human skin melanoma A-375 cells. Biomed Pharmacother 59: S293-S297, 2005.

40. Lai GH, Zhang Z and Sirica AE: Celecoxib acts in a cyclooxygenase 2-independent manner and in synergy with emodin to suppress rat cholangiocarcinoma growth in vitro through a mechanism involving enhanced Akt inactivation and increased activation of caspases-9 and -3. Mol Cancer Ther 2: 265-271, 2003. 